\title{
Le " parlé-chanté » dans Phèdre de Jean Racine et Partage de midi de Paul Claudel
}

\section{Introduction}

Les Anciens [...] distinguaient trois sortes de chants, qu'ils nommaient voix continue quand on parle, voix intermédiaire, quand on lit des vers héroïques ou quand on déclame, voix séparée par des intervalles, quand on chante, ce qui s’appelle encore unité mélodique (Tack, $2001: 111$ ).

Comment la forme hybride du " parlé-chanté » apparait-elle spécifiquement dans Phèdre de Jean Racine et Partage de midi de Paul Claudel ? Telle est la problématique que nous nous proposons d'aborder dans cet article.

Pour ce faire, notre réflexion se divisera en trois parties. Tout d'abord, il s'agira, dans la première partie, de définir ce qu'il faut précisément entendre par le "parlé-chanté ». À quoi cette notion renvoie-t-elle précisément et est-elle liée au Sprechgesang schönbergien ? Tandis que la deuxième partie concernera Phèdre de Jean Racine, la troisième et dernière partie se focalisera sur Partage de midi de Paul Claudel. Comment les protagonistes de la tragédie racinienne et du drame claudélien se trouvent-ils à la lisière du parlé et chanté ? Comment ces deux dramaturges se positionnent-ils face aux discours de leur temps? Telles sont les questions que nous nous posons et auxquelles nous allons tenter de répondre.

Marine Deregnoncourt - candidate à l'École Doctorale en Langues et Lettres de l'Université Catholique de Louvain. Adresse pour correspondance : B. - Place Blaise Pascal, 1, 1348 Louvain-la Neuve (Belgique); e-mail : marianni1@skynet.be 


\section{Le " parlé-chanté ", l'équivalent du Sprechgesang schönbergien?}

En regard de la voix parlée, la voix chantée est davantage mise sur un piédestal mais cette distinction est bien plus complexe qu'il n'y paraît au premier abord. Le parlé-chanté attesté dans le Pierrot lunaire d'Arnold Schoenberg témoigne de cet entredeux entre la voix chantée et la voix parlée. Dès lors, quels liens unissent ces deux types de voix ? Hormis la signification métaphorique, quel sens nous est-il permis de définir (Grosjean, $2001: 71$ ) ?

Arnold Schoenberg met au jour léquivocité de la voix en développant la Sprechstimme, type particulier de récitation à la lisière entre le parlé et le chanté. Ce concept met en exergue deux tensions :

- la parole articulée et les soupirs ou les cris, qui imprègnent le discours structuré de marques émotives et corporelles ;

- la voix-chant et la voix-parole. La musicalisation enrichit la voix-parole d'une grille technique précise quant aux durées, aux hauteurs sonores, aux timbres des instruments et aux voix typiques telles que l'alto ou la soprano.

La Sprechstimme peut être ainsi « analysée comme une mise à l'épreuve des frontières de quatre registres qui s'entrecroisent en elle » (Tack, 2001 : 113), à savoir :

- leffet de sémantisation dû au langage ;

- l'effet de communication intersubjective et de corporéité généré par la parole ;

- le chant en tant quartificialisation de la parole par une voix-instrument « technique »;

- l'impératif de lécriture des mots, de la notation musicale ou d'un texte muet devant être vocalisé.

Pour représenter le monde, l'homme doit nécessairement passer par le signe linguistique, lequel est biface et comprend un signifiant et un signifié. La musique, quant à elle, relève d'une autre instance représentative. Tandis que le langage verbalise « le rapport de représentation symbolique " (Tack, 2001 : 114) qui conjoint l'indice et le sens, la musique serait davantage axée sur la sensibilité. Comment dès lors établir un rapport entre le langage censé référentialiser et la musique liée à un artefact sensible?

Hormis le rythme qu'ils ont en commun, c'est davantage un phénomène d'attraction/répulsion qui lie le texte à la musique. Le signifiant et le signifié distinguent généralement ces deux systèmes macro-sémiotiques. Tandis que le langage verbal transmet une signification, la musique ne dit rien et na donc pas de face signifiée. Pour autant, elle nest ni vide de sens, ni de contenu émotionnel. Par conséquent, la musique peut exprimer ce dont la parole est incapable et, à ce titre, est intrinsèquement liée à la défaillance langagière. 
En tant quart composite, le théâtre est un terrain propice aux échanges musico-littéraires. En attestent les deux parties suivantes de notre article.

\section{Le " parlé-chanté " dans Phèdre de Jean Racine}

Il nous faut d’emblée préciser qu’en 1677, soit quand Jean Racine rédige Phèdre, la «Querelle des Anciens et des Modernes » fait rage (Bloch-Lainé, 2016 : 45'35"). Par cette ultime tragédie profane, le dramaturge tend à démontrer la suprématie de la tragédie sur l'opéra, lequel est qualifié de " tragédie en musique » et est défendu par les Modernes (idem). Avec son ami Nicolas Boileau, Jean Racine est davantage partisan des Anciens. Autrement dit, tous deux défendent l'idée selon laquelle la création artistique nécessite obligatoirement d'imiter les Anciens (Louvat-Molozay, Piéjus, 1995 : 763).

Un des enjeux de la «Querelle des Anciens et des Modernes » est précisément l'opéra car c'est un art moderne et une tragédie entièrement chantée que les Modernes assimilent à la tragédie grecque car toutes deux sont caractérisées par le chant et la danse. Dans ce cadre, Jean Racine entend réaffirmer la supériorité de la tragédie parlée sur la tragédie lyrique.

En 1674, il rédige d'ailleurs Iphigénie en réponse à l'Alceste de Philippe Quinault et Jean-Baptiste Lully, qui ont l'audace de s'emparer d'un sujet du tragique grec Euripide. En regard, Phèdre sert à réaffirmer les valeurs morales tragiques. Lart tragique cherche à exprimer, de manière réglée ${ }^{1}$, le dérèglement. La violence passionnelle tend à devenir davantage verbale et à s'apparenter à un affrontement psychologique (Louvat-Molozay, Piéjus, 1995 : 763).

Dans ce contexte, Phèdre fait ici l'objet d'une analyse métathéâtrale, autrement dit d'une lecture (auto)réflexive du langage tragique. La voix incestueuse et luxurieuse de cette héroïne mythique ne s'apparenterait-elle pas à celle de la Muse, inspiratrice de la tragédie ? La reine athénienne n'allégoriserait-elle pas le théâtre ? Phèdre n'attesterait-elle pas des limites de la tragédie et de « la forme achevée des noces entre la tragédie et le lyrisme » (ibid. : 768) ? Le suicide de cette héroïne mythique, lequel est la coda tragique ${ }^{2}$, ne démontrerait-il pas à fortiori la mort du genre théâtral confronté à un point de non-retour qu'est l'opéra?

Le statut théâtral de Phèdre est ambigu. Tout d'abord, même si la voix atteste conventionnellement de la présence physique des acteurs, les protagonistes raciniens refusent d'apparaître sur scène. Ensuite, ils se complaisent dans un mutisme assumé. Comment un protagoniste théâtral peut-il sciemment se dérober au regard

1. Nombre imposé d’actes, de vers et de syllabes (l’alexandrin à rimes plates). Bloch-Lainé, 2016 : $48^{\prime} 05^{\prime \prime}$.

2. "Mesures finales qui allongent la conclusion d'une pièce musicale ». Louvat-Molozay, Piéjus, $1995: 768$. 
des autres ? Pourquoi refuse-t-il de s'exprimer ? Pour quelle raison le silence fait-il de la parole tragique une «non-parole» ?

D’entrée de jeu, Hippolyte entend quitter le devant de la scène :

Le dessein en est pris : je pars, cher Théramène,

Et quitte le séjour de l'aimable Trézène 3 . (vv. 1-2) (Delmas, Forestier, 1995 : 35).

Il en va de même pour l'entrée de Phèdre en scène : «Soleil, je te viens voir pour la dernière fois » (ibid. : 172). Par conséquent, les protagonistes raciniens se trouvent sur une scène précédant la "vraie" scène et dans un no man's land théâtral. Le non-scénique semble dès lors être la scène sur laquelle se déroule l'action. Cette dénégation théâtrale, qui apparaît dans ce cache-cache entre ombre et lumière, néchappe d'ailleurs aucunement aux confidents des protagonistes qui sont intrigués par ce " pas de deux » équivoque entre avant-scène et avant-jeu.

S'il existe une représentation, elle apparaît sous forme dénoncés performatifs dans lesquels l'espace scénique ne possède plus d’autre référant que les va-et-vient et chassé-croisé des personnages. En effet, les répliques des comédiens sont centrées sur leur entrée et leur sortie de scène. Dès lors, le spectacle se fissure à travers des visions intermittentes et l'apparition et la disparition des protagonistes qui ne parviennent pas à rester longtemps ensemble, n'arrivent à s'exprimer quà travers deux portes et leurs rencontres se font en coulisses. "Rien n'est vu, tout s'entrevoit ; rien n'est dit, tout s'entredit ou [...] s'interdit» (Pot, 1993 : 161). En atteste le rendez-vous manqué et furtif d'Hippolyte et Aricie. Quant à Thésée, il demeure, jusqu'au vers 827 (soit jusquà la moitié de la tragédie), le centre manquant des discours des personnages. Par conséquent, l'existence des protagonistes est fantomatique.

$\mathrm{Au} X \mathrm{XVII}{ }^{\mathrm{e}}$ siècle, représenter est un verbe lié à l'imitatio. Il cherche à reproduire une image ou l'héritage d'une tradition textuelle. Or, dans le théâtre racinien, tout se déroule comme si les protagonistes refusaient de revêtir leur personae dramatis, autrement dit leur rôle et de réactualiser scéniquement des exempla, c'est-à-dire des données mythiques telles quelles sont fournies par la tradition. Phèdre remet ainsi en question la flamboyante scénographie baroque ${ }^{4}$. Les personnages paraissent non seulement refuser d'assumer leur rôle mais apprécient aussi de le pervertir ou plus précisément de léluder (eludere: déjouer).

L'hérö̈ne racinienne parle de l'origine de sa propre voix. En tant qu'actrice qui entend inventer et jouer « son propre texte ", elle souhaite atteindre l'inaccessible limite d'une représentation qui ne s'apparente pas à la répétition du déjà-dit. Les règles étant implicites quant à la façon de déclamer les vers, lacteur bénéficie d'une liberté totale. En atteste l'aveu fantasmatique de Phèdre à Hippolyte (Acte II, Scène 5) dans le-

3. Ladjectif « aimable» concerne Aricie et non pas la ville de Trézène. Pot, $1993: 160$.

4. Le déploiement fastueux de la parole, la spectacularité des lieux, lévocation de légendes et de monstres, la descente aux Enfers sont corrélés à la pastorale amoureuse. Pot, 1993 : 172. 
quel la reine athénienne substitue le fils au père (Louvat-Molozay, Piéjus, 1995 : 768). Soudain, la stratégie de lénonciation change la scène du mythe et l'oblitère en tant que scène et mythe amoureux. Ce travestissement des rôles interroge le changement épistémologique de la notion même de littérature. Cest désormais sur une déconstruction rhétorique du discours qu'une " nouvelle rhétorique ", basée sur l'autonomie lyrique de la voix, peut se construire. Cette «nouvelle rhétorique » est inversée, fait et défait le discours et se conçoit autant pro que contra la rhétorique (ibid. : 764).

À travers une rhétorique inversée, le désenchantement de la parole dans Phèdre ne s'apparenterait-il pas, in fine, à un enchantement dans lequel le langage deviendrait séduction sensuelle du chant et du spectacle ? Le faste baroque de Phèdre, tragédie de " désir de regard » et de " regard de désir » ne rend-t-il pas compte d'un audelà inaccessible de la parole tragique que cache la fascination refoulée de l'opéra ou du théâtre musical (Pot, $1993: 172)$ ?

Car en définitive, Phèdre est une tragédie du langage. La monstruosité opératique sert à révéler le cas-limite du langage où le non-figurable peut, par l'aveuglement des mots, être donné à voir. Dans l'opéra, l'image de la rhétorique tragique ne souvret-elle pas à l'existence phénoménale spectaculaire que lui assure, en faisant fi du langage, la magie machinique et musicale?

En refusant d'accorder à la musique, qui est étroitement liée à la poésie, tout pouvoir de signification comme son efficacité réside plutôt dans des associations d'idées que dans des pensées abstraites, la mimésis aristotélicienne est ainsi dépassée. Est désormais privilégiée une poétique du merveilleux et du spectaculaire, laquelle sera d'ailleurs caractéristique ultérieurement des opéras ramistes. En faisant fi de la puissance des mots et du pouvoir du langage, cette poétique chercherait à atteindre les sens plutôt que le sens.

\section{Le " parlé-chanté » dans Partage de midi de Paul Claudel}

Paul Claudel réfléchit sur le rôle de la musique au théâtre ${ }^{5}$. Où faut-il situer la frontière entre « la parole » et « la musique » ? Même si la musique est fréquemment considérée comme un langage autonome, cette opposition demeure complexe. En effet, la parole et la musique contiennent toutes deux un élément sonore. Partage de midi parait esquisser un imaginaire de la diction spécifique. En effet, cette œuvre réinvente la différenciation entre le parlé et le chanté et tente ainsi délaborer sa propre poétique.

Pour rédiger cette pièce de théâtre, Paul Claudel s'est basé sur Tristan et Isolde de Richard Wagner. D’un point de vue du contenu, il s'agit, dans l'un et l'autre cas, d'un amour absolu. En témoigne ces propos d'Ysé (Acte II) :

5. «Lécroart, Pascal, Paul Claudel et la rénovation du drame musical, Mardaga, Liège, 2004; Plourde, Michel, Paul Claudel, une musique du silence, Presses de l'Université de Montréal, Montréal, 1970 ; Samson, Joseph, Paul Claudel, poète-musicien, Milieu du monde, Genève, 1947 ». Anger, 2011 : 10. 
Ce que nous désirons, ce n’est point de créer, mais de détruire, et que ah ! Il n'y ait plus rien d'autre que toi et moi, et en toi que moi, et en moi que ta possession, et la rage, et la tendresse, et de te détruire et de nêtre plus gênée par ces vêtements de chair. [...]. Ah ! Ce n'est point le bonheur que je t’apporte, mais ta mort, et la mienne avec elle (Antoine, $2012: 93)$.

Ce désir d'amour total, d'osmose, de fusion et de dépassement de l'individualisme «toi/moi », lequel génère inévitablement la mort, est formulé dans le cimetière de Hong Kong. Cette séquence, subséquente à la rencontre des deux protagonistes sur un bateau en partance pour la Chine, est un moment clé au cours duquel l'adultère entre Ysé et Mesa va être crûment organisé. En effet, à la demande de son amante, Mesa envoie cyniquement De Ciz (l’époux d'Ysé) à la mort. Remarquons d'ailleurs que la jeune femme a précédemment affirmé à son mari qu'elle le tromperait, s'il devait la quitter (Acte II, scène 1) : « Je sens en moi une tentation ».

La requête amoureuse d'Ysé est vraisemblablement parallèle à la conception wagnérienne de l'amour. Ce sentiment vise à rechercher l'unité et la compréhension totale de l'autre. Lamour fait donc fi de l'individuation, échappe aux contraintes sociales et aux limites corporelles, physiques et se réalise uniquement dans la mort.

Toutefois, Paul Claudel se distancie du grand Tout (Gesamtkunstwerk), caractéristique de la métaphysique wagnérienne. Ysé oppose le corps à l'âme. La fusion des «âmes » introduit une coupure binaire au sein du sujet humain. Lâme immatérielle de cette jeune femme permet la relation intensément érotique à l'autre. Le corps demeure un lieu d'opacité, d'interaction, de désir et de souffrance.

Ces présupposés permettent d'éclairer la fin de Partage de midi. Mesa a refusé la fusion d'amour total proposée par Ysé. Il ne s'est donc pas donné à elle et n'a pas souhaité s'ouvrir à l'altérité. C'est pourquoi elle l'a quitté pour Amalric (son ancien amant). Refusant d'accepter cette situation, Mesa va chercher à la récupérer ainsi que leur enfant. S'engage alors une lutte à mort avec Amalric au cours de laquelle Mesa périt. Cependant, l'état corporel de ce dernier demeure trouble et obscur. Est-il ressuscité ou est-ce un fantôme?

La scène théâtrale devient dès lors un espace au-delà de la mort, un " entre-deux » qui scelle l'union entre deux âmes incarnées et douées de parole. Le mode d'expression choisi pour témoigner de cette incertitude est la musique (dépourvue de partition). Paul Claudel intitule d'ailleurs l'avant-dernière scène de Partage de midi : "Le Cantique de Mesa ». "Le reste du drame exprime le contrepoint, le mariage de ces deux âmes qui se donnent l'accord l'une à l'autre, à l'intérieur de la Musique de Dieu, qui est Silence et Harmonie » (Plourde, 1970 : 176).

Dans Partage de midi, la manifestation de la " parole » claudélienne répond à une poétique caractérisée par la métaphore musicale. En témoignent les appellations telles que « leitmotif », " duo d'amour » ou " mélodie » pour qualifier la prosodie textuelle. Paul Claudel s'octroie en fait à son propre compte la locution mallarméenne : " reprendre à la musique son bien » (Locatelli, 2001 : 15). Selon Stéphane Mallarmé, 
la poésie est la Musique même. Au cœur de la " querelle du vers libre ", Paul Claudel, aux côtés de Stéphane Mallarmé, s'oppose à René Ghil, partisan de l'instrumentalisation du verbe (idem). D’une part, le dramaturge entend dé-référentialiser le langage par des répétitions ou des différences et revenir ainsi aux fondamentaux du signe (tel que ce concept sera défini par Ferdinand de Saussure, père de la linguistique moderne). Entre 1880 et 1935, par la prégnance du modèle musical, les artistes comprennent que les arts peuvent se passer de mots. D'autre part, dans Partage de midi, Paul Claudel entend insister sur la puissance imageante de l'autre, autrement dit réintégrer, à l'intérieur des mots, la puissance évocatrice des images, des illustrations et des monstrations d'affectivité desquelles la musique rend compte au sein de lopéra ou de la symphonie.

Cependant, au-delà de la prégnance «musicale » propre au texte claudélien, lequel propose en lui-même l'idée d'un texte musical, les didascalies différencient nettement ce qui relève du chant de ce qui relève de la parole. Ainsi, à l'intérieur même de l’emploi des mots considérés comme de la "musique », il y aurait des moments musicaux et d'autres qui ne le seraient pas. En outre, bien qu'il ne renseigne pas comment prononcer les vers de Partage de midi, le dramaturge accorde une grande importance au ton. Les personnages " déclament " ou " récitent ». "Réciter " revient à s'effacer devant lénonciation et à refuser dêtre énonciateur. Il en va de même pour « déclamer » à la différence près que ce verbe est emphatique et insiste sur la spectacularité de la parole.

Le «chant » est toujours corollaire d'une parole de conversion de Mesa et d'Ysé. Quelle que soit sa modalité sonore, en regard des paroles qui ont « repris à la musique, son bien ", le "chant» établit un «chant nouveau " (Anger, 2011:16). Les personnages tiennent eux-mêmes un discours extrêmement précis sur la voix, la parole et la musique. Lors de la dernière scène, Ysé reprend à son compte la métaphore romantique de la voix en tant quaccession spirituelle à son être profond et intime : " Ne te tais point, mon bien-aimé [...] que jaillisse et m’entende avec mon propre son d'or pour oreilles / Commencer, affleurer comme un chant pur et comme une voix véritable à ta voix ton éternelle Ysé » (Antoine, 2012 : 149).

Par ailleurs, le texte de Partage de midi oppose la voix au silence. Au premier acte, Mesa dit à Ysé qu'il s'est tenu devant Dieu comme un homme désireux de tout donner mais dépourvu de parole. Au troisième acte qui se déroule exclusivement durant la nuit, Mesa entend «sauver » sa bien-aimée et lui propose de fuir à ses côtés. Il lui reproche son manque de réaction qu'il ne va pas pouvoir supporter et va vouloir commettre une tentative de meurtre, empêchée in extremis par Amalric (l'amant d'Ysé) qui finira par le tuer. Face au mutisme de sa bien-aimée, Mesa l'accuse de surdité. Il s’agit bien là de la capacité d’audition et non de la parole. Mesa établit ainsi une «théorie langagière et sonore». Le son s'oppose au silence. La surdité, le silence, l'altérité et la non-réponse sont autant d'épreuves fondamentales et nécessaires pour atteindre l'amour et la rédemption. Ysé ne prononce pas un mot, reste silencieuse et accepte autant la mort de Mesa que sa propre mort. Passer par le silence et la rup- 
ture totale est, pour elle, la condition sine qua non pour ne pas revenir à cette relation d'amour possessif que lui propose Mesa, lequel est soucieux de conquérir l'univers : "ô jaurais voulu tout voir, avoir avec appropriation " (Antoine, 2012 : 49). Simultanément, passer par le silence, la déréliction et la mort permet au « je » de séprouver en tant que " je », en symbiose avec une instance divine dont il tire son existence. Le silence et la surdité deviennent alors une écoute excessivement forte. Ce n'est plus un gouffre mais une présence. Mesa, «l'homme qui sait » (ibid. : 167), entend cette femme de l'intérieur, telle une question ou une parole dénuée de bruit. Lamour génère ce silence et cette intériorisation de la parole, qui progressivement se résorbe au sein du silence créateur de Dieu. Mesa comprend alors que ce nest pas une parole qu'il a ouïe mais un regard et une voix au fond de ses entrailles. La parole témoigne d'une réalité fuyante car le témoignage est inutile quand lon détient la réalité elle-même.

Par conséquent, le silence est biface. Il est autant surdité quécoute maximale, lieu de meurtre et de mort que lieu de la pleine prise de conscience du « je » dépendant d'une ascendance divine. Tel est le nœud qui scelle la damnation à la rédemption. C'est précisément dans cet «entre-deux » que le « chant » peut advenir. Dans ce cadre, la musique demeure bel et bien une métaphore. Le son est un des médiateurs entre le logos et le caractère concret du monde. La dichotomie entre la parole et la musique repose sur l'énonciation. En effet, Paul Claudel oppose le langage quotidien au langage de l'art et de la conversion.

La représentation de Partage de midi pose directement la question sonore de la voix et de la façon dont un individu va, avec sa voix et son physique, prononcer les mots. Or, cette manière de dire les vers n’est aucunement renseignée par Paul Claudel. Le "Cantique de Mesa » est un long monologue qui, au sein de l'action, n’a aucune fonction. Mesa ne fait que méditer sur les évènements antérieurs.

La scission entre la parole et le " chant » correspond à une rupture relative au statut corporel de l'acteur sur scène. L'identité corporelle de Mesa est trouble puisqu'il est décédé. L’arrivée ultérieure inopinée d'Ysé, en transe hypnotique, relève également d'un statut corporel atypique. Le « chant» sous-tend non seulement les affects des deux protagonistes mais met aussi en exergue leur ambiguïté corporelle. Le "Cantique " témoigne de cet entre-deux corporel et examine profondément la notion même d'incarnation. Sur scène, le comédien incarne un rôle et une parole "musicalisée " pour satisfaire le but poétique mallarméen repris par Paul Claudel. Si l'acteur parvient à éprouver la parole émise et à établir un lien entre son être le plus intime et l'équivocité de son personnage présentifié et unifié et simultanément autre que lui-même, il peut alors ressentir ce « chant nouveau » suggéré par Mesa et Ysé.

Partage de midi s'apparente à un mélodrame informel dans lequel la musique constitue l'arrière-fond de la parole (Locatelli, 2001 : 94). Paul Claudel souhaite travailler sur l'incarnation de la réalité et de la vérité. Il cherche à retranscrire une parole qui est, dans l'instant de la représentation, un accompagnement sonore. 
En définitive, le dramaturge refuse d'essentialiser la musique mais accepte, par contre, que la représentation théâtrale soit accompagnée de musique. Il considère son théâtre comme un tout englobant une partie du traitement sonore et redéfinit ainsi, sans pour autant annuler l'oxymore, les deux termes opposés "parole» / «musique ». $\mathrm{La}$ "parole » absorbe intégralement la « musique », qui inclut le ton, le chant (sans hauteur fixe) et tout type d'accompagnement instrumental. Le "sonore " peut parfaitement prolonger la parole. Toutefois, cette " parole " doit renfermer l'idée d'une articulation entre le (dis)continu, le son et le silence, le mot et le corps, le sublime et le trivial. Incarner pareille tension à travers une parole unifiée est à recréer sans cesse au cours d'une nouvelle mise en scène.

\section{Conclusion}

Comment la forme hybride du "parlé-chanté » apparait-elle dans Phèdre de Jean Racine et Partage de midi de Paul Claudel ? Telle est la problématique que nous nous sommes proposés d'aborder dans cet article.

Tout d'abord, nous avons défini le "parlé-chanté ». Cette acception est liée à la Sprechstimme schönbergienne qui atteste de cette frontière poreuse entre le parlé et le chanté. Littéralement, il s'agit d'un parler sous forme musicale. Lambiguïté est ainsi d'emblée posée. Dans les deux parties suivantes, nous avons tenté de démontrer que les protagonistes des deux pièces de théâtre se trouvent à la lisière du parlé/ chanté. Nous avons pu constater une prégnance opératique manifeste. L'influence musicale est donc attestée chez ces deux dramaturges qui (dé)jouent les codes et se positionnent face aux discours de leur temps.

\section{BIBLIOGRAPHIE}

Anger V. 2011. Lopposition parlé-chanté dans Partage de midi de Paul Claudel. In Freixe G. \& Porot B. Les interactions entre musique et théâtre. Reims. Université d’Amiens-Reims. 10-25. Antoine G. 2012. Paul Claudel. Partage de midi. Paris. Gallimard.

Bloch-Lainé V. Jean Racine, le caméléon (1639-1699). URL : http://www.franceculture.fr / emissions/une-vie-une-oeuvre/jean-racine-le-cameleon-1639-1699, consulté le 01/08/2016.

Delmas C., Forestier G. 1995. Racine. Phèdre. Paris. Gallimard.

Grosjean M. 2001. Le jeu musical des voix dans l'interaction. In Badir S. \& Parret H. Puissances de la voix, corps sentant, corde sensible. Limoges. Presses universitaires de Limoges. 71-90.

Locatelli A. 2001. Littérature et musique au XXe siècle. Paris. PUF.

Louvat-Molozay B., Piéjus A. 1995. La petite musique de Jean Racine. À la recherche de la musicalité du texte racinien. XVII siècle 189. 762-768.

Plourde M. 1970. Paul Claudel : une musique du silence. Montréal. Presses Universitaires de Montréal.

Pot O. 1993. Phèdre ou le suicide de la tragédie. Travaux de littérature VI. 159-172. 
Tack L. 2001. L'entre-deux de la voix. Questions à propos de la Sprechstimme dans Pierrot lunaire de Schoenberg. In Badir S. \& Parret H. Puissances de la voix, corps sentant, corde sensible. Limoges. Presses universitaires de Limoges. 111-130.

\section{The " parlé-chanté " in Phèdre to Jean Racine and Noon's Sharing to Paul Claudel}

ABSTRACT: In this article we will seek to answer how, in Phèdre to Jean Racine and Noon's Sharing to Paul Claudel, the "parlé-chanté » is particularly significant. To do so, this article will be divided in three parts. In the first part, the "parlé-chanté » will be defined and linked to Arnold Schoenberg's Sprechgesang. While the second part will issue on Phèdre by Jean Racine, the third and final part will focalize on Paul Claudel's Noon's Sharing. By focusing on the two aforesaid plays we will try to prove that the operatic voice is always present in the acting choices and that the two playwrights accustomed the dramatic rules of their time.

Keywords: The "parlé-chanté », The operatic voice, Phèdre and Noon's Sharing. 